\title{
A SIMPLE PROOF OF THE THEOREM OF TORELLI BASED ON TORELLI'S APPROACH
}

\author{
ALBERTO COLLINO
}

\begin{abstract}
We give a simple proof of the classical theorem of Torelli, based on Torelli's original approach and on the use of Poincaré's formulas.
\end{abstract}

The original theorem proved by Torelli [7] is more general than the modern reader expects, it says in an equivalent formulation: Let $C$ be a nonsingular curve of genus $g$, let $J$ be the Jacobian variety of $C$, let $W$ be the standard divisor on $J$; if $f: G \rightarrow J$ is a morphism from a curve $G$ of genus $g$ such that (a) $f(G)$ generates $J$ as a group and (b) degree $f^{*} W=g$, then (i) $f$ is an injection, (ii) $(J(C), W(C))=(J(G), W(G))$, and (iii) $G$ is obtained from $C$ by a transformation of the first or second species.

We recall that classically a transformation of first species on an abelian variety means a translation, while a transformation of second species is a translation followed by the involution which sends each element to its opposite.

The Matsusaka-Ran criterion greatly extends (i) and (ii); it says: Let $(A, X)$ be a principally polarized abelian variety of dimension $g$, let $f: G \rightarrow A$ be a map from a curve $G$ to $A$; if (a) $f(G)$ generates $A$ as a group and (b) degree $f^{*}(X)=g$, then $(A, X)=(J(G), W(G))$ and $f$ is one of the standard inclusions. A simple proof of the Matsusaka-Ran criterion is given in $[\mathbf{3}]$.

The original formulation of the theorem of Torelli follows therefore from the Matsusaka-Ran criterion and the usual form of

(1) "Torelli" Theorem. If $(J(C), W(C))=(J(G), W(G))$, then $G$ and $C$ are isomorphic curves and $G$ is obtained from $C$ via a transformation of first or second species on $J$.

Several proofs of (1) are known (cf. [2]), based on different techniques and points of view; our purpose here is to show that the approach of Torelli gives a simple proof of (1). We believe that the original proof of Torelli was somewhat complicated because he was aiming at the more general result he proved.

Our proof is characteristic free. Matsusaka in [4] showed that Torelli's arguments could work also in positive characteristic. To accomplish this he had to construct for its own use new pieces of the theory of abstract algebraic varieties. In a way our proof is also a simplification of Matsusaka's proof, but we make essential use of two results which have been proved by him in positive characteristic: (1) Poincaré's formulas, which I learned from Mattuck [6], and (2) the Riemann

Received by the editors March 10, 1986.

1980 Mathematics Subject Classification (1985 Revision). Primary 14K05, $14 \mathrm{H} 40$.

The author was partially supported by NSF Grant DMS-84-02209, by C.N.R., and by the Italian Ministry of Public Education. 
singularity theorem to the effect that: (a) the singular locus $\Sigma$ of the theta division $W(C)$ is the locus of the classes of special divisors of degree $(g-1)$ and (b) $\operatorname{dim}(\Sigma) \leq g-3, \operatorname{dim}(\Sigma)=g-3$ if and only if $C$ is hyperelliptic (this is contained in or can be deduced in a standard way from the appendix of [4]).

We start be recalling some general facts on curves. The contemporary reference is [1]; a reader looking for characteristic free proofs should refer to [5].

We assume in the following that $C$ is a nonsingular algebraic curve over an algebraically closed ground field. For any positive integer $d$ we denote by $C[d]$ its $d$-fold Cartesian product and by $C(d)$ its $d$-fold symmetric product. The points of $C(d)$ represent the positive divisors of degree $d$ on $C$; if $\mathbf{a}$ is such a divisor, we will also let a denote the corresponding point of $C(d)$. We fix a reference point $p_{0} \in X$; there are maps $i: C(n-1) \rightarrow C(n), i(\mathbf{a})=\mathbf{a}+p_{0}$. We denote by $C(n-j)+p_{1}+\cdots+p_{j}$ the image of the inclusion $\mathbf{b} \rightarrow \mathbf{b}+p_{1}+\cdots+p_{j}$. $J(C)$ or simply $J$ is the Jacobian variety of $C$. There is a map $u_{n}: C(n) \rightarrow J$ where $u_{n}\left(n p_{0}\right)$ is the identity of $J, u_{n}$ is sometimes called the Abel-Jacobi map: $u_{n}(\mathbf{a})=\operatorname{class}\left(\mathbf{a}-n p_{0}\right)$. The divisor $W(C)$, or simply $W$, is $u(C(g-1))$. This divisor is uniquely determined up to a translation; the couple $(J(C), W(C))$ is called the polarized Jacobian of $C$.

We denote $W_{d}:=u_{d} C(d), 0 \leq d \leq g$, so that $W_{g-1}=W$; we write simply $C$ instead of $W_{1}$. Let $R$ be the ring of cycles on $J$ modulo numerical equivalence, let $w_{d}=\operatorname{class}\left(W_{d}\right)$, Poincaré's formulas are $w^{m}=m ! w_{(g-m)}$. The group operation $\mu: J \times J \rightarrow J$ induces the Pontrjagin product $\mu_{*}: R \times R \rightarrow R$, there are formulas $\mu_{*}\left(w_{n}, w_{m}\right)=\left(\begin{array}{c}n+m \\ n\end{array}\right) w_{n+m}, n+m \leq g$.

We denote by $S$ the locus of special divisors in $C(g)$, namely $S:=\{\mathbf{a}:(K-$ a) is effective $\}$; here $K$ is the canonical divisor class. Let $U:=u_{g}(S), U$ is obtained from $W_{(g-2)}$ by a transformation of second species, hence class $U=w_{(g-2)}$. Let $k=$ $u_{2 g-2}(K)$. We write $j: J \rightarrow J$ for the involution $j(x)=k-x$; note $j(W)=W$ and $U=j\left(W_{(g-2)}\right)$. If $M$ is a subset of $J$ and $a \in J$ we write ${ }_{a} M:=\{(a+m): m \in M\}$ and ${ }^{-} M:=\{(-m): m \in M\} . \Sigma$ is the singular locus of $W$; if $C$ is not hyperelliptic then $\operatorname{dim} \Sigma<g-3$, if $C$ is hyperelliptic then $\Sigma$ is a translation of $W_{(g-3)}$.

We write $W(p)=u_{g}\left(C_{(g-1)}+p\right)$, so that $W(p)={ }_{\left(p-p_{0}\right.} W$. We have $W(p) \cap W=$ ${ }_{\left(p-p_{0}\right)} W_{(g-2)} \cup U, p \neq p_{0}$, and in fact $W(p) \cdot W={ }_{\left(p-p_{0}\right)} W_{(g-2)}+U$, as a product of cycles, because of Poincaré's formulas.

Let $A$ be a curve in $W$, let $\alpha=\operatorname{degree}(A \cdot W)$ computed in $R$.

(2) Lemma. (i) $A \cap{ }_{\left(p-p_{0}\right)} W_{g-2} \neq \varnothing, A \cap U \neq \varnothing$, (ii) if $A \cap \Sigma=\varnothing$ then $\alpha=\operatorname{degree}\left(A \cdot{ }_{\left(p-p_{0}\right)} W_{(g-2)}\right)_{W}+\operatorname{degree}(A \cdot U)_{W}$.

Proof. (i) It suffices to check $A \cap_{\left(p-p_{0}\right)} W_{g-2} \neq \varnothing$ for al! $\eta \in C$, then $j(A \cap U)=$ $j(A) \cap W_{g-2} \neq \varnothing$ so that $A \cap U \neq \varnothing$. By assumption $A$ is the image of a curve $A^{+}$in $C_{(g-1)}$. Any curve in $C(g-1)$ intersects the locus $C(g-2)+p$ because any curve in $C[g-1]$ intersects the divisor there which is the pull back of $C(g-2)+p$.

(ii) By assumption $A$ is supported in the nonsingular locus of $W$ and we may use the projection formula

$$
\alpha=\operatorname{degree}(A \cdot W(p))_{J}=\operatorname{degree}\left(A \cdot{ }_{\left(p-p_{0}\right)} W(g-2)\right)_{W}+\operatorname{degree}(A \cdot U)_{W} .
$$

The last fact we need to recall is that

$$
\left\{x \in J:{ }_{x} C \subset W\right\}=W_{g-2} .
$$


ProOF. One inclusion is clear. The other one amounts to saying that given a divisor class $\alpha$ of degree $(g-2)$ which is not effective, there is a point $p \in C$ such that $\alpha+p$ is still not effective. By hypothesis the linear system $|K-\alpha|$ has dimension 0 , hence there is a point $p$ such that $K-\alpha-p$ is not effective.

PROOF OF (1).

By hypothesis, class $W(G)=w$, hence

$$
\operatorname{class}(G)=w^{(g-1)} /(g-1) !
$$

in $R$.

The starting point of Torelli is the question about how many points $U$ and a general translate of $G$ which meets $U$ have in common.

Let $\delta: J \times J \rightarrow J$ be the difference map $\delta(x, y)=x-y$; we write $Z=\delta(U \times G)$. Namely

$$
Z=\left\{z \in J:{ }_{z} G \cap U \neq \varnothing\right\}
$$

and we write $s: U \times G \rightarrow Z$ as the restriction of $\delta$. From Poincaré formulas and the formulas for the Pontrjagin product we have

$$
\text { class } Z=((g-1) / \sigma) w,
$$

where $\sigma$ is the degree of $s$. Note that $\sigma$ divides $(g-1)$, because $w$ is not a divisible class in $R$.

The second question of Torelli is about the number of points $p \in C$ such that ${ }_{z} G \subset W(p)$ when there is at least one such $p$. We define

$$
V(p):=\left\{x \in J:{ }_{x} G \subset W(p)\right\} .
$$

Since $W(p)$ is a translation of $W(G)$, then $V(p)$ is a translation of $W_{g-2}(G)$; hence class $V(p)=w^{2} / 2$. From (2) we have that $x \in V(p)$ implies

$$
{ }_{x} G \cap U \neq \varnothing, \quad \text { i.e. } V(p) \subset Z \text {. }
$$

Let $F:=\{(x, p): x \in V(p)\} \subset J \times C$. We know that the projection to $J$ maps $F$ to $Z$; let $f: F \rightarrow Z$ be the induced map. The degree $\beta$ of $f$ means (naifly at least for the moment) the numbers of points $p$ such that ${ }_{z} G \subset W(p)$ when ${ }_{z} G \cap U \neq \varnothing$. Similarly the degree $\sigma$ of $S: U \times G \rightarrow Z$ is $\operatorname{card}\left(U \cap{ }_{z} G\right)$.

We are goint to prove (1) $\beta=\sigma$ and (2) the naif interpretation of $\sigma$ and $\beta$ is correct, i.e. both $f$ and $s$ are separable maps and $U$ and ${ }_{z} G$ intersect transversally in $\sigma$ distinct points for general $z \in Z$.

Let $E:=\{(y, p): y \in W(p)\} \subset J \times C$, the family of $W(p)$ divisors. The following diagram is useful:

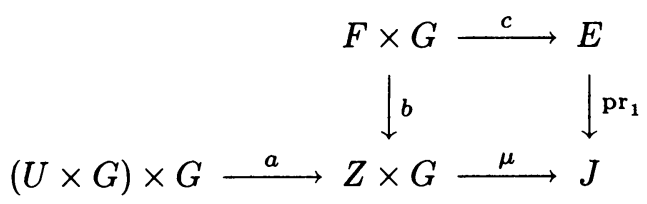

where $a=(s, \mathrm{id}), b=(f, \mathrm{id}), c((x, p), q)=(x+q, p)$.

Since $V(p)=W_{(g-2)}(G)$, then $V(p) \times G \rightarrow W(p)$ is separable of degree $g-1$, hence $c$ is separable of degree $g-1$. Also $\operatorname{pr}_{1}: E \rightarrow J$ is a separable map, of degree $g$. It follows that $b$, equivalently $f$, is a separable map. By Poincaré's formulas, 
degree $(\mu a)=g(g-1)$; therefore $\sigma=\operatorname{degree} a=\operatorname{degree} b=\beta$, and $\operatorname{card} f^{-1}(z)=\sigma$ for a general point $z \in Z$.

We show now that $s: U \times G \rightarrow Z$ is a generically smooth map, so that $s^{-1}(z)=$ $\left\{\left(x_{1}, y_{1}\right), \ldots,\left(x_{\sigma}, y_{\sigma}\right)\right\}$ for a general point $z \in Z$ with $x_{1} \neq x_{j}, i \neq j$, and $T_{x_{i}, U} \cap$ $T_{y_{i}, G}=0$, i.e. ${ }_{z} G$ and $U$ intersect transversally in $\sigma$ points.

We identify in the usual way the tangent spaces $T_{x, U}, T_{y, G}$ with subspaces of $T_{e, J}, e$ the identity of $J$. The differential at $(x, y)$,

$$
d s: T_{x, U} \oplus T_{y, G} \rightarrow T_{e, J},
$$

is the restriction of the difference map on $T_{e, J} ; d s$ is not injective if and only if $T_{y, G} \subset T_{x, U}$. Now the canonical image of $G$ in $\mathbf{P}^{g-1}$ is also the image of $G$ in $\mathbf{P}\left(T_{e, J}\right)$ via the Gauss map $y \rightarrow \mathbf{P}\left(T_{y, G}\right)$, therefore it is not contained in any linear subspace of $\mathbf{P}^{g-1}$. If $x$ is a nonsingular point of $U$, then $G \not \subset \mathbf{P}\left(T_{x, U}\right)$; hence there is a point $y \in G$ for which the differential $d s$ is injective at $(x, y)$.

Everything proved for $G$ holds also for ${ }^{-} G$. There is the variety $Z^{\wedge}:\{z \in J: z=$ $\left.u-x ; u \in U, x \in{ }^{-} G\right\}$ and, for a general point $y \in Z^{\wedge},{ }_{y}\left({ }^{-} G\right)$ and $U$ intersect transversally in $\sigma^{\wedge}$ points, further $\sigma^{\wedge}$ divides $(g-1)$. By using the involution $j$ we see that this means that the general translate of $G$ which meets ${ }_{\left(p-p_{0}\right)} W_{g-2}$ meets it transversally in $\sigma^{\wedge}$ points.

If $\operatorname{dim} \Sigma<(g-3)$, i.e. if $C$ is not hyperelliptic, then one has ${ }_{y} G \cap \Sigma=\varnothing$ for a general point $y$ with the property ${ }_{y} G \subset W$. We use (2) with $A={ }_{y} G ; \sigma+\sigma^{\wedge}=g$ and both $\sigma$ and $\sigma^{\wedge}$ divide $g-1$. Hence, up to a permutation, $\sigma=g-1$ and $\sigma^{\wedge}=1$. Since $\sigma=\beta$, then given a general $z \in Z$ there are distinct points $p_{1}, \ldots, p_{g-1}$ with ${ }_{z} G \subset W\left(p_{i}\right)$. Now $\operatorname{dim} U=g-2$, hence in general ${ }_{z} G \not \subset U$ so that ${ }_{z} G$ lifts birationally to a curve, $G^{+}$say, in $C(g)$. $G^{+}$is contained in $C(g-1)+p_{i}$, $i=1, \ldots, g-1$, therefore $G^{+}$is the curve $C+\left(p_{1}+\cdots+p_{g-1}\right)$ in $C(g)$ and $G$ is its image in $J$.

If $\operatorname{dim} \Sigma=g-3$, then both $G$ and $C$ are hyperelliptic curves and $\Sigma=W_{(g-3)}(G)$ up to translation, moreover $G={ }_{x}\left({ }^{-} G\right)$ for some $x \in J$.

Let $z \in Z$ be a general point, let $p \in C$ with $_{z} G \subset W(p)$, let $\Sigma(p)$ be the singular locus of $W(p)$. From the inclusion $\Sigma(p) \subset U \subset W(p)$ we obtain a commutative, but not cartesian, diagram

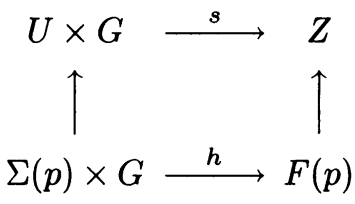

where $F(p)=s(\Sigma(p) \times G)$ and $h$ is the restriction of $s$. The map $h$ is, up to translation, the ordinary $W_{(g-3)}(G) \times G \rightarrow W_{(g-2)}$, hence degree $h=g-2$. Since $\sigma \geq$ degree $h$ and $\sigma$ divides $(g-1)$ then $\sigma=(g-1)$. The proof is now the same as for the nonhyperelliptic case.

\section{REFERENCES}

1. E. Arbarello, M. Cornalba, P. Griffiths, and J. Harris, Geometry of algebraic curves, Vol. 1, Springer-Verlag, New York, 1985.

2. A. Beauville, Le problème de Torelli, Séminaire Bourbaki no. 651, Novembre 1985. 
3. A. Collino, A new proof of the Ran-Matsusaka criterion for Jacobians, Proc. Amer. Math. Soc. 92 (1984), 329-331.

4. T. Matsusaka, On a theorem of Torelli, Amer. J. Math. 80 (1958), 784-800.

5. A. Mattuck, Symmetric products and Jacobians, Amer. J. Math. 83 (1961), 189-206.

6. _ , On symmetric products of curves, Proc. Amer. Math. Soc. 13 (1962), 82-87.

7. R. Torelli, Sulle varietà di Jacobi, Rend. R. Accad. Lincei 22 (1913), 98-103.

DiPARTIMENTO Di MATEMATICA, UNIVERSITÀ DI TORINO, 10123 TORINO, ITALY 